\title{
Meeting Report \\ Use of Alternative Methods: From Fundamental to Industrial Research
}

doi:10.14573/altex.1812172

On October 31, 2018 the first 3Rs Open Day took place at the University of Milano - Bicocca. The Open Day was supported by the Earth and Environmental Science Department, University of Milano - Bicocca, by MISTRAL (Integrated Models for Prevention and Protection in Environmental and Occupational Health) University of Brescia Research Centre, and by the Carlo Pesenti Foundation.

The relevance and novelty of the initiative was highlighted by the Rector of the University of Milano-Bicocca, Maria Cristina Messa, who opened the day. She spoke about both the educational content of the 3Rs Open Day and its cultural implications. There is a growing consciousness at the university and among its scholars that ethics is fundamental in all fields of research. Considerable efforts are made by the university in this direction through the commitment and efforts of the ethical committee and the work and collaboration with delegates of the European Commission. In this context, the need to move from the concept of $3 \mathrm{Rs}$ to that of $4 \mathrm{Rs}$ (Reduction, Refinement, Replacement and Responsibility) was also stressed.

The history of the 3Rs and their significance was introduced by Laura Gribaldo (European Commission). In 1959, the British academics Rex Burch and William Russell proposed a model for researchers to become more aware of the suffering that animal experimentation causes its experimental subjects. The 3R principle refers to three approaches to reduce this suffering: replace, reduce, and refine. Key aspects a scientist must weigh when planning an animal experiment are: the validity of the scientific data that can be obtained; the transferability of this data to humans; and the degree of suffering inflicted on experimental animals. Ethical committees must conduct a cost-benefit analysis for each proposal to perform animal experimentation.

Francesca Pistollato (European Commission) introduced in vivo and in vitro models in neurosciences and discussed their potential, limits, and applications. In vitro models promise to improve the efficacy of drug discovery as well as human risk assessment, and help identify the chemicals' mechanisms of action. Human stem cell-derived neuronal models, cultured as monolayers (2D) or in 3D complex structures, together with microfluidics approaches and co-culture systems, which include physiological nervous system barriers (e.g., the blood-brain barrier or the choroid plexus) have been developed. While these in vitro models are still under development, their use, complemented with in silico, in chemico, and existing in vivo and epidemiological data, may already support a paradigm shift towards the use of human cell-based in vitro methods in modern neuroscience.
An example of reduction was presented by Chiara Urani (University of Milano - Bicocca and MISTRAL Research Centre) in the field of carcinogenesis. The bioassay on rodents still represents the gold standard and currently no stand-alone method is able to fully replace animal testing for carcinogenicity assessment. After a brief history of the in vitro cell transformation assays (CTAs), the core methodological procedures of CTAs and the morphological evaluation of the endpoint (foci of transformed cells) were described. These assays find broad use by academia, industry, environmental protection agencies, and reference laboratories (e.g., EURL ECVAM) for fundamental research, screening, and validation studies. Major gaps to be filled include i) understanding of the mechanisms of chemical carcinogenesis; ii) development of more in vivo-like 3D models; iii) automatic classification of transformed foci. The current CTAs can be used as a component of an integrated approach to testing and assessment (IATA).

Arti Ahluwalia (University of Pisa and Director of 3R Centre) presented the newly constituted 3R Center, established in December 2017 between the University of Pisa and the University of Genoa, with the aim of promoting the principles of the 3Rs in teaching and research. The presentation then discussed advanced in vitro methods, especially the potential of and development challenges faced by microfluidic systems. These systems still require further development to achieve reliable models that fully mimic in vivo processes.

Integrated testing strategies (ITS) and their application to protect public health were presented by Giovanna Mazzoleni (University of Brescia and Director of the MISTRAL Centre). Examples of application of ITS in environmental toxicology were presented, i.e., the EXERA and the water challenge projects detect estrogenic compounds in wastewaters subjected to various treatments and the MAPEC Life project assesses the toxic/carcinogenic potential of very fine particulate matter (PM 0.5).

A representative of ETT S.p.A. Genoa, Susanna Alloisio, showed the point of view of industry in the context of environmental monitoring regarding advanced in vitro methods and presented neurotoxicity measurements of microalgal biotoxins in seawater. The innovative assays are based on electrophysiological recordings measured on a rat neuronal network grown on multi-electrode arrays (MEA). Advantages of the MEA approach are a functional readout, high sensitivity, high-content information, and improved versatility. The approach will be used to compile a database on the toxicological effects of different algal species for use in risk evaluation during the periodic monitoring of sea environments. 
Practical experience of applying the 3Rs principles to animal experimentation was described by Guido Cavaletti (University of Milano - Bicocca, President of the Animal Welfare Body). He described the administrative processes and documents that challenge researchers working in the field of pre-clinical studies as well as the long process and sometimes unsuccessful efforts to develop an alternative method to the point where it can be submitted to validation bodies (e.g., EURL ECVAM).

In silico methods were the central topic of the afternoon, opened by Roberto Todeschini, Davide Ballabio and Francesca Grisoni (University of Milano - Bicocca, Milano Chemometrics and QSAR Research Group). A brief introduction to the quantitative structure activity relationship (QSAR) strategy, based on physical-chemical properties and related biological activities of molecules, was given. Different case studies and collaborative projects were presented to better explain areas of applicability of QSAR strategies, including the large-scale project "Predictive models for acute oral systemic toxicity", which has potential application in regulatory frameworks, the "Collaborative Estrogen Receptor Prediction Project (CERAPP)", and the "Collaborative Modelling Project of Androgen Receptor Activity (CoMPARA)". The presentations highlighted how QSAR approaches represent a rapid and inexpensive way to prioritize chemicals, and that the combination of multiple QSAR predictions improves the reliability of the outcome.

Laura Bonati and Maurizio Bruschi (University of Milano - Bicocca) presented advanced in silico methods to investigate molecular mechanisms associated with toxicity effects. In silico modeling of molecular properties provides insight into a protein's biological functions, as well as into the effects of changes induced by physiological and/or environmental factors. "Molecular docking" investigates protein-ligand binding at molecular level. Three docking case studies were presented: a) the aryl hydrocarbon receptor, which mediates toxicity induced by many environmental contaminants; b) hypoxia inducible factor $2 \alpha$, a transcription factor that is a possible target of anti-tumor agents; and c) the pregnane $\mathrm{X}$ receptor, a nuclear receptor target of drugs. Also, the general principles of the molecular dynamics approach and its applications in the study of conformational changes of proteins due, for example, to site-specific mutagenesis and drug/contaminant-protein interactions were explained. As a case study, an investigation of the long-range conformational effects induced by the binding of DNA to the p53 onco-suppressor protein was presented.

The final part of the Open Day was characterized by a round table on "The business future working with alternative methods" in which representatives from academia and industry described their personal and working experiences and gave an idea of the future opportunities for careers involving alternative methods, especially addressed to the young students and researchers.

\section{Chiara Urani ${ }^{1,2}$, Maurizio Bruschi ${ }^{1}$, Sara Casati $^{3,4}$ and Laura Gribaldo 5}

${ }^{1}$ Department of Earth and Environmental Sciences, University of Milano - Bicocca, Italy; ${ }^{2}$ MISTRAL University Research Centre, University of Brescia, Italy; ${ }^{3}$ Research Division, University of Milano - Bicocca, Italy; ${ }^{4}$ BBMRI-ERIC, Graz, Austria; ${ }^{5}$ Chemical Safety and Alternative Methods Unit (F.3), Directorate F-Health, Consumers and Reference Materials, European Commission, Ispra (VA), Italy 